\title{
Determinação do dano em pavimentos asfálticos por meio da combinação do modelo S-VECD com análises elásticas
}

\author{
Lucimar da Silva Santiago ${ }^{1}$, Samuel de Almeida Torquato e Silva ${ }^{2}$, Jorge Barbosa Soares ${ }^{3}$ \\ 1Universidade Federal do Cariri, Juazeiro do Norte-CE, Brasil, lucimar.santiago@ufca.edu.br \\ ${ }^{2}$ Laboratório de Mecânica dos Pavimentos, Universidade Federal do Ceará, Fortaleza-CE, Brasil, samuel@det.ufc.br \\ ${ }^{3}$ Laboratório de Mecânica dos Pavimentos, Universidade Federal do Ceará, Fortaleza-CE, Brasil, jsoares@det.ufc.br
}

\section{Recebido:}

10 de agosto de 2017

Aceito para publicação:

17 de agosto de 2017

Publicado:

31 de agosto de 2018

Editor de área:

Francisco Thiago Aragão

\section{Palavras-chaves:}

Dimensionamento,

Pavimentos asfálticos,

Fadiga,

Dano.

\section{Keywords:}

Design,

Asphalt pavements,

Fatigue,

Damage.

DOI:10.14295/transportes.v26i2.1446

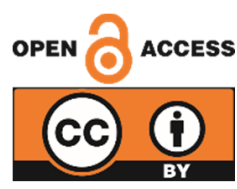

\begin{abstract}
RESUMO
A previsão do desempenho dos materiais em campo é um dos desafios da pavimentação. Essa previsão passa pela geração de modelos matemáticos que relacionem a caracterização dos materiais em laboratório e o desempenho destes mesmos materiais em estruturas reais. Sendo o dano por fadiga um dos principais defeitos estruturais nos pavimentos asfálticos brasileiros, o objetivo do trabalho é apresentar uma metodologia de previsão desse defeito utilizando análise estrutural elástica-linear, tendo como dado de entrada a evolução do dano por fadiga obtida por meio de ensaios laboratoriais em materiais asfálticos. Sabendo-se da importância da temperatura e da velocidade na via para o desempenho dos pavimentos quanto ao dano por fadiga, esses dois parâmetros também são considerados numa análise simplificada. Ao final, é apresentado um exemplo prático dos principais passos da metodologia sugerida, a qual é devidamente consolidada em um programa computacional. A metodologia proposta é um passo para o meIhoramento da correlação entre o campo e o laboratório, facilitando o processo de predição do comportamento dos materiais asfálticos em pavimentos reais.
\end{abstract}

\begin{abstract}
Performance prediction of materials in the field is one of the challenges in pavement engineering. Such prediction requires mathematical modeling that relates materials characterization in the laboratory and the performance of these same materials in actual structures. Since fatigue damage is one of the main distresses in Brazilian asphaltic pavements, the objective of this work is to present a methodology to determine such distress using linear elastic analysis, having as inputs fatigue damage evolution parameters obtained in laboratorial tests. Given the importance of temperature and vehicle speed in roadways for pavement performance with respect to fatigue damage, these two parameters are also considered in the simplified analysis. At the end, it is presented a practical example with the main steps of the developed methodology, which is programmed in a specific pavement design software. The proposed methodology is a step in enhancing field-laboratory correlation, facilitating the behavior prediction of asphaltic materials in real pavements.
\end{abstract}

\section{INTRODUÇÃO}

O Atualmente o Brasil se encontra em um momento singular da história da sua pavimentação rodoviária: o desenvolvimento, no âmbito da Rede Temática de Asfalto, com o apoio da Petrobras e o envolvimento de diversas universidades e do DNIT, de um método de dimensionamento de pavimentos asfálticos baseado em princípios mecanísticos e com o suporte de uma base de dados de campo sem precedentes no país. Um dos aspectos relevantes desse método é a sua construção com o suporte técnico-científico de instituições de diferentes estados e também de fora do país. Além disso, dados de vias obtidos antes, durante e após as respectivas execuções foram coletados em diferentes regiões do Brasil. Essas informações foram inseridas em um Banco de Dados (Sistema da Rede Temática de Asfalto, SRTA), um passo 
essencial para a criação de métodos mecanístico-empíricos com equações de previsão de desempenho tão fidedignas quanto possível ao comportamento dos materiais em campo.

Os defeitos estruturais mais evidentes nas vias com pavimentação asfáltica são deformação permanente e trincamento, sendo este último resultante, principalmente, da fadiga dos materiais. Assim, neste trabalho, apresenta-se uma metodologia analítica para a determinação do dano por fadiga, que combina o modelo S-VECD (Nascimento, 2015) com análises elásticas. Os resultados serão utilizados nos passos subsequentes de um framework computacional ora em desenvolvimento, a ser calibrado para análises de previsão de área trincada ao longo da vida útil de um pavimento. Esses passos não são, no entanto, foco do presente artigo, que visa neste momento avaliar a viabilidade de uso do modelo S-VECD (Simplified Viscoelastic Continuum Damage) combinado com a elasticidade linear, o que representa a sua contribuição científica. Para o fim proposto são usadas caracterizações mecânicas de misturas asfálticas aplicadas em trechos monitorados no Brasil, com as quais se realiza a simulação da evolução do dano dessas misturas numa determinada estrutura. As simulações de dano foram realizadas por meio do programa CAP 3D-D, que é um desdobramento do programa de elementos finitos CAP3D (Holanda et al., 2006), constituindo-se num programa de dimensionamento (daí adicionar-se -D) de pavimentos asfálticos.

\section{FUNDAMENTAÇÃO TEÓRICA}

\subsection{Métodos de dimensionamento de pavimentos mecanístico-empíricos}

Os métodos de dimensionamento mecanístico-empíricos utilizam equações que preveem o desempenho da estrutura do pavimento ao longo de sua vida útil, algo importante não somente do ponto de vista estrutural, mas também da análise de viabilidade econômica de diferentes períodos de projeto (Mello et al., 2016). Essas equações são desenvolvidas e aperfeiçoadas por meio dos resultados de observações de campo. Esse é um dos principais desafios desses métodos, e requer um amplo e consistente banco de dados de caracterização de materiais e pavimentos. A análise estrutural e os modelos de previsão de desempenho são centrais nos métodos mecanístico-empíricos, que são constituídos por três subgrupos principais: dados de entrada, análise (estrutural e de desempenho) e dados de saída. Os dados de entrada necessários são: tráfego ao qual o pavimento é submetido, condições ambientais da região (no mínimo a temperatura ambiente), informações das camadas e seus materiais constituintes (e.g., no caso do revestimento, o Módulo de Resiliência ou Módulo Dinâmico, espessura, coeficiente de Poisson, curva de fadiga do material).

Após a inserção dos dados de entrada prossegue-se com a análise estrutural do sistema de camadas, que pode ser elástica-linear, não linear, viscoelástica, entre outras. Pode estar fundamentada em métodos analíticos ou numéricos (e.g., elementos finitos), cada um com vantagens e restrições. A metodologia de análise pode ser em dois passos, quando tem-se uma resposta estrutural (tensões e deformações) e esta serve de dado de entrada para a análise de previsão de desempenho do pavimento ao longo de sua vida útil, ou seja, o dano que ele sofre ao longo do tempo. Já outras metodologias, especialmente as mecanicistas mais avançadas (e.g., FlexPAVETM 1.0, antigo LVECD e atualmente em teste pela FHWA), fazem isso em um único passo (NCSU, 2014). De posse dos resultados de simulação do comportamento do material em campo, o projetista avalia se a previsão de vida útil do pavimento, quanto aos defeitos considerados (\% de área trincada, afundamento da trilha de roda, etc.) atende aos valores admissíveis estipulados para cada tipo de defeito.

A previsão da vida de serviço do pavimento é, todavia, um dos maiores desafios dos engenheiros de pavimentação (Kim, 2009). É esta lacuna que os modelos de desempenho procuram preencher utilizando parâmetros conhecidos das camadas do pavimento. Segundo Huang (2004), eles são o elo fraco nos métodos mecanístico-empíricos, pois requerem uma extensiva calibração e verificação de dados de campo para serem confiáveis. Neste sentido, o presente estudo busca contribuir para utilização do dano por fadiga como passo intermediário para a construção de modelos de desempenho úteis a um método nacional de dimensionamento de pavimentos asfálticos. 


\subsection{Dano por fadiga em misturas asfálticas}

\subsubsection{Breve introdução ao dano por fadiga}

Dano, segundo Lemaitre (1996), é a deterioração que ocorre no material antes da falha, geralmente não podendo ser visto ou tocado. Para Kim (2009), dano é a perda de resistência de um material provocado por algum mecanismo de falha (trincamento por fadiga ou deformação permanente). 0 foco do presente estudo é no dano por fadiga.

Do ponto de vista da mecânica do contínuo, dano, provocado em termos de trincamento, é dado por $\mathrm{D}=\mathrm{SD} / \mathrm{S}$, ou seja, densidade efetiva de microdefeitos. Onde SD é a área efetivamente trincada e $\mathrm{S}$ é a área total analisada (Lemaitre, 1996). Para Shah et al. (1995), a mecânica do dano é dirigida para a obtenção de índices que descrevem a redução da capacidade funcional de estruturas, em termos de ciclos de carga, cuja equação básica é representada por:

$$
E_{\text {dan }}=E_{\text {orig }}(1-D)
$$

onde:

$\mathrm{E}_{\mathrm{dan}}=$ rigidez do material danificado;

$\mathrm{E}_{\text {orig }}=$ rigidez do material original (não danificado);

$\mathrm{D}=$ dano (entre 0 e 1 , onde $\mathrm{D}=0$ significa que o material não está danificado e $\mathrm{D}=1$ que ele está danificado).

Na pavimentação, no entanto, o dano por fadiga é frequentemente expresso como uma taxa que é dada pela relação entre o número de repetições de carga acumulado e o número de repetições de carga admissível (Equação 2). 0 número admissível de repetições de carga em um dado período e condição de análise $\left(N_{f i}\right)$ é associado à resposta (tensão ou deformação de tração) do pavimento neste período e condição. Na Lei do dano acumulado de Miner, consideram-se diferentes períodos "i" (meses ou estações do ano), sendo o dano final (D) um somatório dos danos ocorridos nestes períodos, como indicado pela Equação 2 (NCHRP, 2004)

$$
\sum_{i=1}^{T} D_{i}=\frac{n_{i}}{N_{f i}}
$$

Onde:

$\mathrm{T}$ = número total de períodos;

$n_{i}=$ tráfego atual para o período " $i$ ", ou seja, tráfego projetado na via num dado período considerado na análise, podendo ser obtido pela Equação 3;

$N_{f i}=$ valor máximo de solicitações de tráfego que determinado material resiste em campo sem sofrer ruptura, num dado período "i" e condições, indicado por deformações sofridas pelo material asfáltico em ensaios laboratoriais. A partir deste ponto referenciaremos $N_{f i}$ por $N_{f}$.

$$
n_{i}=\frac{N\left(1-(1+t / 100)^{p}\right)}{1-(1+(t / 100))}
$$

Onde:

$\mathrm{N}$ = número de eixos padrões considerado no projeto da via no ano inicial de abertura ao tráfego, por pista considerada na análise;

$\mathrm{t}=$ taxa de crescimento anual do tráfego;

$\mathrm{P}=$ período correspondente $\mathrm{a}$ análise (em meses).

$\mathrm{O}$ valor de $\mathrm{N}_{\mathrm{f}}$ pode ser determinado por diferentes funções, sendo atualmente mais utilizada a Equação 4 (NCHRP, 2004), do Asphalt Institute. 0 modelo utilizado nos trechos experimentais da Ilha do Fundão-RJ é uma variação deste, sendo representado pela Equação 5. Para Huang (2004), no entanto, o importante não é a função de transferência usada, mas a sua calibração cuidadosa, na qual se aplica um 
fator campo-laboratório (shift factor) adequado (à região), permitindo que a equação de previsão corresponda às observações de campo.

$$
\begin{gathered}
N_{f}=K_{1}\left(\frac{1}{\varepsilon_{t}}\right)^{K_{2}} E^{K_{3}} \\
N_{f}=K_{1}\left(\varepsilon_{t}\right)^{K_{2}}
\end{gathered}
$$

onde: mento;

$\varepsilon_{\mathrm{t}}=$ deformação principal de tração, tradicionalmente considerada na fibra inferior do revesti-

$\mathrm{E}=$ rigidez da mistura asfáltica no revestimento;

$\mathrm{K}_{1}, \mathrm{~K}_{2}$ e $\mathrm{K}_{3}=$ coeficientes de regressão laboratorial, inerentes a mistura asfáltica.

Conforme mencionado, o dano simulado D varia de 0 a 1 (i.e., 100\%) e as funções de transferência convertem este parâmetro em área trincada observada em campo (\%AT). Em termos práticos, esse índice leva em consideração a extensão total do trecho analisado e a área considerada por técnicos como trincada. 0 parâmetro \%AT não será abordado neste trabalho, sendo a sua relação com o dano objeto de trabalho subsequente dos autores.

\subsubsection{Trabalhos nacionais envolvendo dano por fadiga}

No Brasil, Nascimento (2015) e Fritzen (2016) desenvolveram funções de transferência relacionando dano por fadiga e \%AT, diferenciando-se na abordagem (viscoelástica e elástica, respectivamente) e nos ensaios mecânicos. Ambos utilizaram dados de campo e de laboratório de trechos monitorados pela Rede Temática de Asfalto, tanto da Ilha do Fundão, Rio de Janeiro, quanto de outros estados do Brasil.

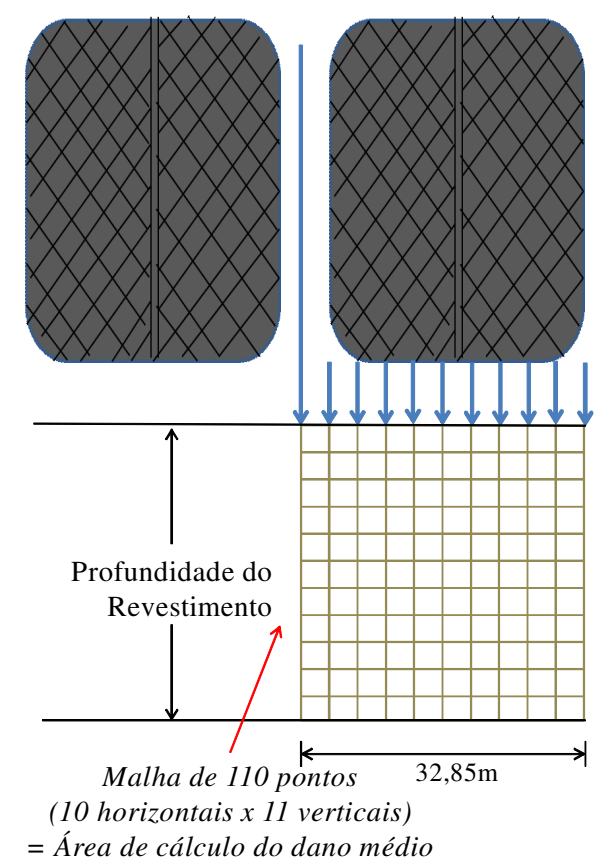

Figura 1. Coordenadas dos pontos de análise da deformação principal máxima proposta por Nascimento (2015)

Nascimento (2015) utilizou o modelo S-VECD, variações de temperatura, velocidade dos veículos, distribuição horária do tráfego, dados de ensaios de $\left|E^{*}\right|$ e de fadiga por tração direta (TD), associada a uma análise viscoelástica. 0 programa para realizar o cálculo estrutural com essas premissas foi o LVECD 
(Layered Viscoelastic Pavement Analysis for Critical Distress), desenvolvido na North Carolina State University, cuja primeira versão foi liberada em 2012 (precursor do atual FlexPAVETM 1.0). Os resultados de resposta estrutural foram utilizados para a determinação do dano médio, que corresponde ao somatório dos danos calculados em cada ponto de análise abaixo de um dos pneus do eixo padrão, dividido pelo número total de pontos, sendo esta uma área do revestimento afetada pelo carregamento. Na Figura 1 é possível verificar essa distribuição de pontos abaixo da carga, que correspondem a uma malha de 10 elementos horizontais e 11 verticais, totalizando 110 pontos de análise.

Em termos gerais, Fritzen (2016) fez uso do mesmo conceito de análise de dano, sendo utilizado o MR e a vida de fadiga por compressão diametral (CD) para caracterizar o revestimento em laboratório. Estes foram associados a uma análise elástica para calibrar e validar, nas suas condições de análise, o modelo proposto por Nascimento. Pelas características da análise, não são consideradas variações de temperatura, de velocidade e distribuição horária do tráfego.

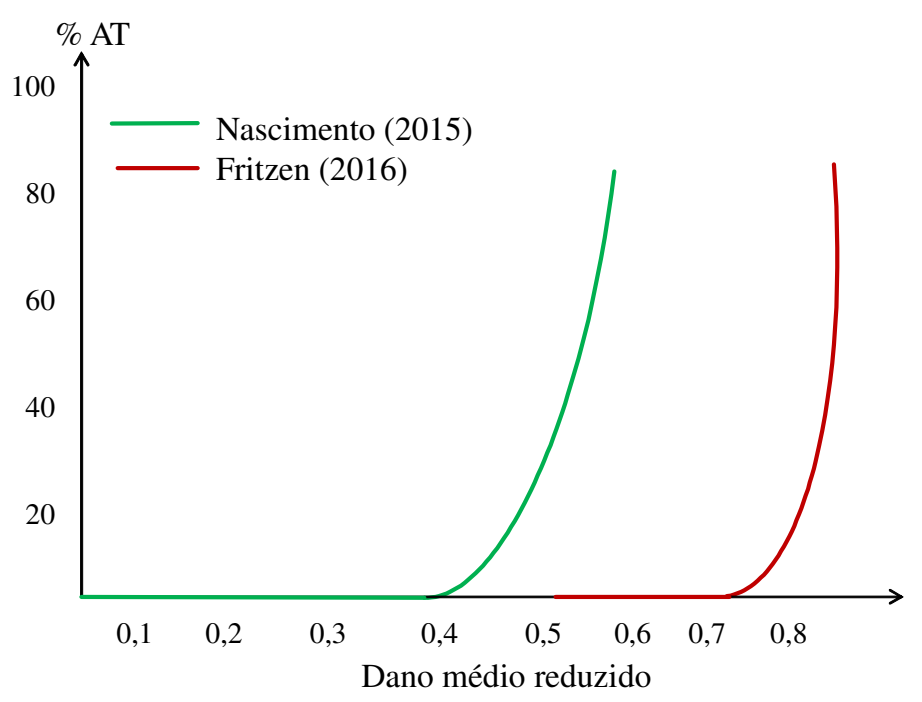

Figura 2. Comparativo entre a Função de Transferência de Nascimento (2015) e Fritzen (2016)

Em ambas as pesquisas se detectou que a melhor forma de determinação do dano era limitando-o entre 0 e 1. Especificamente no que diz respeito a correlação dano por fadiga e \%AT, Nascimento (2015) aplicou um fator de deslocamento de dano às curvas de dano médio (que têm um mesmo formato), de modo a correlacioná-lo ao início do trincamento em campo. Esse trincamento começa em níveis de dano diferentes, a depender da taxa de crescimento do dano. Tal fato levou Nascimento (2015) a desenvolver a função de deslocamento de dano, que cria um espaço de dano reduzido no qual todos os pavimentos apresentaram as mesmas correlações entre dano reduzido e área trincada. A mesma estratégia foi usada por Fritzen. A Figura 2, adaptada de Fritzen (2016), apresenta um gráfico com as curvas simuladas de \%AT x dano médio reduzido em cada uma dessas pesquisas. Em Fritzen (2016) a curva está mais próxima do valor de dano máximo (igual a 1) do que no resultado de Nascimento (2015). Dado que as misturas utilizadas nas duas pesquisas são as mesmas, são dignos de nota como possíveis causas da diferença: o método de análise estrutural (elástico-linear x viscoelástico), o tipo de dado de entrada do revestimento, i.e., rigidez (MR x $\left.\left|E^{*}\right|\right)$ e vida de fadiga (CD x TD). Sobre as diferenças entre esses ensaios podem ser consultados os trabalhos de Babadopulos $(2013,2015)$ e de Nascimento $(2015)$.

\section{METODOLOGIA DE DETERMINAÇÃO DO DANO POR FADIGA DE MISTURAS ASFÁLTICAS}

\subsection{Aspectos preliminares}

A fim de testar, preliminarmente, a influência do tipo de parâmetro de rigidez e do ensaio de vida de fadiga nos resultados de dano, fez-se, para uma mesma mistura e temperatura, sob uma análise elástico- 
linear, simulações computacionais, ora utilizando-se como dados de entrada o par MR e CD, ora o par $\left|E^{*}\right|$ e TD. A Figura 3 apresenta os resultados desse teste e mostra a diferença de comportamento em relação ao dano médio quando se utiliza cada par. Investigar essa diferença é uma das motivações da presente pesquisa, que traz uma contribuição efetiva ao adaptar a metodologia utilizada pelos pesquisadores referenciados no item 2.2.2, para a determinação do dano por fadiga em misturas asfálticas. As premissas consideradas para as análises neste estudo são delineadas a seguir:

- Aplicando-se a teoria da elasticidade, o parâmetro de rigidez das misturas asfálticas adotado será o $\left|\mathrm{E}^{*}\right|$, obtido da curva mestra do material, conforme temperatura e frequência determinadas a partir da espessura do revestimento, da temperatura do ar e da velocidade de projeto da via;

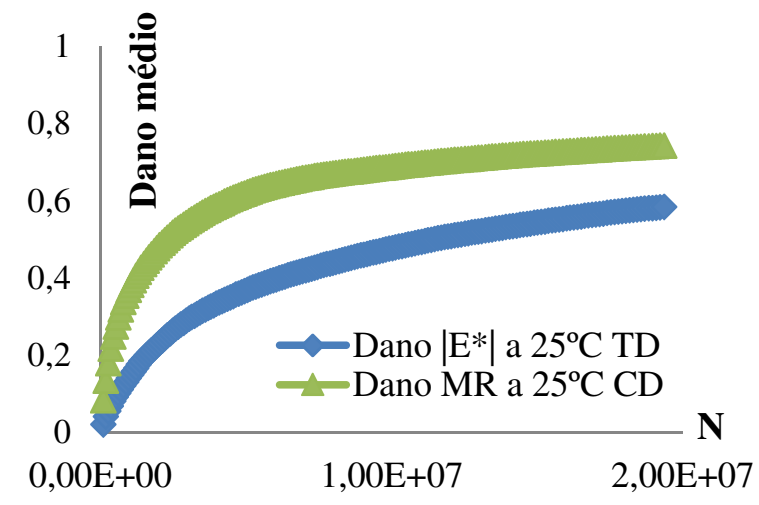

Figura 3. Efeito do uso de diferentes parâmetros de rigidez e vida de fadiga

- Por meio do ensaio de TD, aplicando-se o modelo S-VECD acoplado ao critério de ruptura GR, baseado na energia dissipada pelo material, realiza-se a caracterização da vida de fadiga da mistura em laboratório (para maiores detalhes, o leitor é referido a Nascimento, 2015);

- Assume-se análise elástico-linear, todavia fazendo uso de um programa de elementos finitos. Torquato e Silva et al. (2013) mostram que é possível chegar a resultados semelhantes em análises elásticas e viscoelásticas, desde que se considere o valor da rigidez do revestimento devidamente influenciado pela temperatura e frequência de carregamento, por meio dos dados de Módulo de Relaxação ou Dinâmico, sendo este segundo adotado no presente artigo;

- Realiza-se a análise do dano em três temperaturas diárias (manhã, tarde e noite) em cada mês do ano. Essa estratégia permite incluir o efeito da temperatura e da velocidade na rigidez das misturas asfálticas na análise elástico-linear. A inclusão desta variável possibilita considerar variações climáticas. Ao final, é apresentado um exemplo em que a análise é realizada com e sem variação de temperatura.

Os detalhes da metodologia proposta, itemizada para facilidade de compreensão, encontram-se nos subitens a seguir.

\subsection{DADOS NECESSÁRIOS À METODOLOGIA}

As informações para a consecução da metodologia proposta são divididas em dados de laboratório e dados de campo.

\subsubsection{Dados de laboratório}

- Parâmetros da curva mestra de $\left|E^{*}\right|$ do revestimento asfáltico, para que variações de temperatura e velocidade da via possam ser consideradas nas análises. 0 coeficiente de Poisson também é um dado de entrada da mistura asfáltica, contudo, será considerado constante; 
- Rigidez das subcamadas, em termos dos respectivos MR (obtidos de ensaios ou por retroanálise) e coeficiente de Poisson. No caso da análise elástica-linear, considera-se um valor único de MR para cada subcamada;

- Parâmetros da curva de vida de fadiga da mistura asfáltica, utilizando os resultados do ensaio de TD (AASHTO TP 107, 2014). Informações mais detalhadas sobre a referida curva constam no item 3.3.

\subsubsection{Dados de campo}

- Data de início da operação da via (mês e ano);

- Tráfego previsto em projeto, transformado em número $\mathrm{N}$ pelos fatores de equivalência do United States Army Corp of Engineers (USACE), tal como em Nascimento (2015);

- Distribuição horária do tráfego, já que se consideram diferentes horários e temperaturas;

- Taxa percentual de crescimento anual do tráfego;

- Espessuras das camadas;

- Dados de clima, i.e., temperaturas médias do ar da região em que a mistura foi aplicada em campo. São consideradas para cada mês do ano as temperaturas médias nos períodos (horas) de 00:00 - 08:00, 08:00 - 16:00 e 16:00 - 00:00.

\subsection{Determinação do $\mathbf{N}_{\boldsymbol{f}}$}

Nascimento (2016) formulou um conjunto de equações que, a partir do modelo S-VECD e do critério de ruptura baseado em GR, permite prever a vida de fadiga $\left(N_{f}\right)$ de misturas asfálticas em função da deformação, da frequência, da parcela do ciclo em tração e da temperatura (Equação 6). Esses parâmetros não dependem de determinações empíricas. 0 parâmetro de dano é determinado pela perda de energia dissipada devido a perda de rigidez do material durante o ensaio de vida de fadiga sob tração direta uniaxial (TD) e aplicando-se o modelo S-VECD. A Equação 6 é mecanisticamente fechada e foi escolhida para a metodologia aqui proposta.

$$
N_{f}=\left(\frac{\delta}{Y\left(\frac{C_{12}}{p}\right)+1}\right)^{\frac{1}{\Delta+1-\frac{C_{12}}{p}}}
$$

onde:

$$
\begin{gathered}
\delta=\frac{1}{2}\left(\varepsilon^{R}{ }_{T A}\right)^{2} C_{11}\left(\frac{p\left(C_{11} C_{12}\right)^{\alpha}\left(\varepsilon_{T A}^{R}\right)^{2 \alpha} k_{1}}{f_{R} 2^{\alpha}}\right)^{\frac{C_{12}}{p}} \\
p=\alpha-\alpha C_{12}+1 \\
G^{R}=Y\left(N_{f}\right)^{\Delta} \\
C(S)=1-C_{11} S^{C_{12}} \\
\varepsilon_{T A}^{R}=\frac{1}{E_{R}} \frac{\beta+1}{2}\left(\left(\varepsilon_{0, p p}\right)_{i}\left|E^{*}\right|_{L V E}\right)
\end{gathered}
$$

onde:

$$
\begin{aligned}
& \mathcal{E}^{R}{ }_{T A}=\text { amplitude da pseudo-deformação; } \\
& \alpha=\text { taxa de evolução do dano; } \\
& \mathrm{C}_{11} \text { e } \mathrm{C}_{12}=\text { coeficientes da curva } \mathrm{C} \times \mathrm{S} ; \\
& \mathrm{k}_{1}=\text { fator de forma da carga; } \\
& \mathrm{f}_{\text {red }}=\text { frequência reduzida; }
\end{aligned}
$$


$\mathrm{G}^{\mathrm{R}}$ = taxa de variação média da energia de pseudo-deformação liberada (por ciclo) durante todo o ensaio;

$N_{f}=$ número de ciclos até a falha;

$\mathrm{Y}=$ coeficientes do critério de ruptura $G^{R}$ vs. $N_{f}$;

$\Delta=$ expoente da equação $Y$;

$\mathrm{C}(\mathrm{S})=$ integridade do material;

$\mathrm{S}$ = parâmetro de dano;

$\varepsilon_{0 . p p}=$ Amplitude de deformação pico a pico;

$\beta=$ parcela do ciclo em que ocorre tensão de tração (um valor usual é de $-0,08$, que na prática significa que ocorre compressão em $80 \%$ do ciclo);

$\left|\mathrm{E}^{*}\right|_{\mathrm{LVE}}=$ módulo dinâmico viscoelástico linear nas condições de temperatura e frequência em questão.

\subsection{Determinação do dano médio}

0 cálculo do dano por fadiga é dividido em duas etapas, descritas adiante: (i) cálculo das respostas (deformação principal máxima de tração) e (ii) cálculo do dano.

\subsubsection{Determinação das deformações principais}

Primeiramente, prossegue-se com o cálculo das deformações principais máximas de tração para inclusão na equação de $N_{f}$. Para tal fez-se uso, na presente metodologia, de uma análise elástica-linear. Diferentemente de métodos nos quais a determinação da deformação é calculada apenas na fibra inferior do revestimento, neste método, similarmente a Nascimento (2015) e a Fritzen (2016), discretiza-se um espaço no revestimento que se situa horizontalmente entre as duas rodas do eixo simples de roda dupla. Como se faz uso do método dos elementos finitos na presente análise, consideram-se 240 pontos de Gauss, contidos em 60 elementos, sendo 30 logo abaixo da carga, conforme Figura 4. Apesar da axissimetria assumida, é possível considerar a roda dupla por meio da superposição de efeitos, pois se trata de um modelo linear.

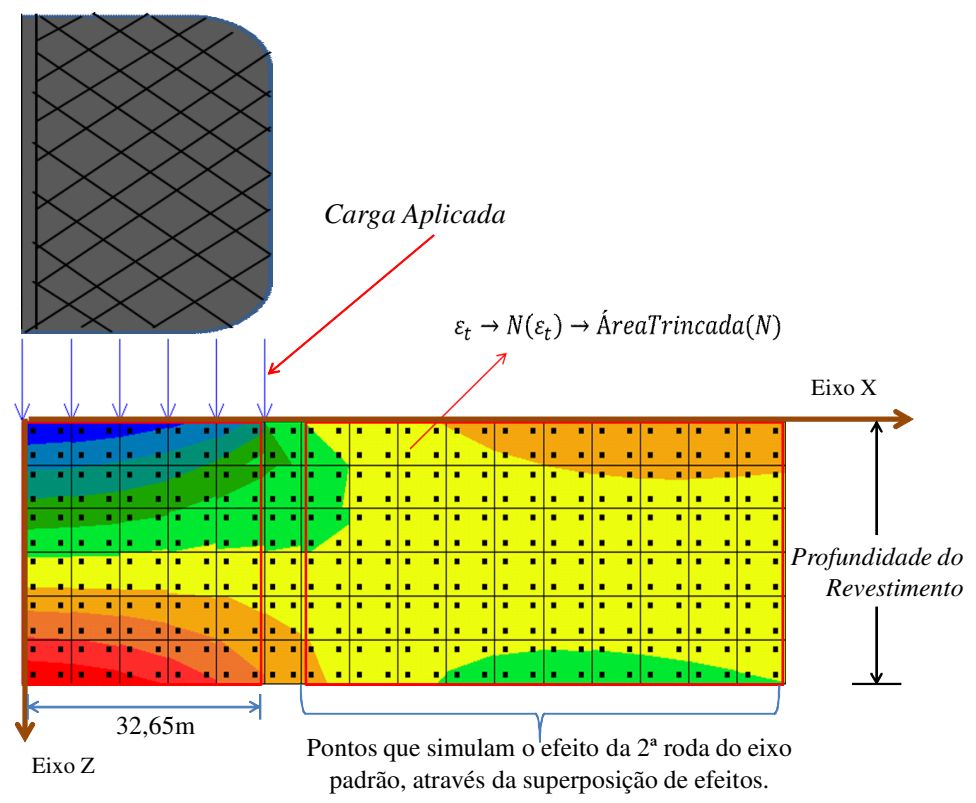

Figura 4. Coordenadas dos pontos de análise da deformação principal máxima utilizada na metodologia apresentada neste artigo 
Realizada a análise estrutural, o cálculo de dano segue com a determinação da deformação principal máxima em cada um dos 240 pontos de Gauss. Apesar da análise não ser viscoelástica, se inseriu nessa metodologia a influência de diferentes temperaturas e a velocidade do carregamento para o cálculo das deformações.

Obs: Se $\varepsilon_{t} \leq 0$, então $\mathrm{D}=0$, ou seja, não ocorreu dano naquele ponto.

\subsubsection{Considerações sobre a temperatura}

Para determinação da temperatura considerou-se o revestimento dividido em três subpartes, nas quais a temperatura foi calculada em seu centro. Para isso, fez-se uso da Equação (7) de estimativa de temperatura no pavimento (Motta, 1991), com base na Pesquisa de Avaliação Estrutural de Pavimentos (PAEP) do IPR/DNER.

$$
T(x)=T_{\text {sup }}+\left(T_{\text {sup }}-T_{\text {ar }}\right)\left(1-e^{(0,06855-0,002633 x) x}\right)
$$

Onde:

$\mathrm{T}(\mathrm{x})=$ Temperatura na profundidade " $\mathrm{x}$ " do revestimento;

$\mathrm{T}_{\text {sup }}=$ Temperatura da superfície do revestimento;

$\mathrm{T}_{\mathrm{ar}}=$ Temperatura do ar próxima ao pavimento.

\subsubsection{Determinação do dano médio}

A determinação do dano é realizada aplicando-se a Lei de Miner (Equação 1) nos 240 pontos de Gauss, nos quais é calculada a média para cada subperíodo de análise. As análises são divididas em meses e em turnos (manhã, tarde e noite). Desta forma, para cada temperatura ou frequência diferente inserida, há uma análise realizada. Tal procedimento fornece uma curva Dano Médio x N. Destaca-se que não é necessário para a aplicação da metodologia o uso de um programa computacional específico, cabendo ao projetista escolher aquele que melhor se adequa a situação. No caso do CAP 3D-D, por exemplo:

- Estão embutidas rotinas de análise estrutural e de dimensionamento em um único programa;

- Realiza-se o cálculo direto do dano e da área trincada ao longo da vida de projeto;

- Considera-se a velocidade de projeto da via em análise;

- Considera-se a distribuição temporal da temperatura, podendo-se chegar a níveis de detalhe de 3 turnos em cada dia do ano, ou mais simplificadamente, 3 turnos em dias padrões que possam ser usados em diferentes estações do ano (o nível de detalhamento desejado pode ser facilmente alterado pelo usuário do programa);

- Considera-se $\left|E^{*}\right|$ do revestimento por meio de parâmetros da curva mestra. Os valores utilizados foram obtidos pelas Equações 8, 9 e 10, que constam em Nascimento (2015) e em Gouveia (2016);

$$
\begin{gathered}
\log \left|E^{*}\right|=a+\frac{b}{1+\frac{1}{e^{d+g * \log \left(f_{R}\right)}}} \\
\log \left(a_{T}\right)=\alpha_{1} T^{2}+\alpha_{2} T+\alpha_{3} T \\
f_{R}=f \times a_{T}
\end{gathered}
$$

onde:

a, b, d, g, $\alpha_{1}, \alpha_{3}$ e $\alpha_{3}=$ coeficientes de regressão das curvas;

$\mathrm{T}=$ temperatura em ${ }^{\circ} \mathrm{C}$;

$f_{R}=$ frequência reduzida;

$a_{T}=$ coeficiente de ajuste da relação tempo-temperatura;

- Considera-se a vida de fadiga do material por meio das constantes do ensaio de fadiga obtidas pelo ensaio de tração direta (TD). Outra alternativa, seria considerar-se por meio das proprida- 
- des das curvas de integridade do método S-VECD, porém isso não é objeto do presente artigo e pode ser encontrado em Nascimento (2015).

\section{EXEMPLO PRÁTICO}

A fim de tornar a metodologia clara, inclui-se um exemplo prático de simulação do dano por fadiga ao longo de um período pré-estabelecido. Apresentam-se as diferenças entre os resultados de dano com e sem a consideração de diferentes velocidades, temperaturas e parâmetros de fadiga. Para isso, foram usados os seguintes dados de entrada, referentes a uma mistura específica. Há de se ressaltar que o comportamento será variável, a depender do tipo de mistura analisada.

- Data de início de operação da via: Jan/2007;

- Tráfego inicial $(\mathrm{N}):$ 1,04E+06;

- Velocidade (km/h): 60 (quando a velocidade não for o parâmetro em análise);

- Taxa de crescimento do tráfego (\%): 3,0;

- Estrutura composta por 4 camadas, todas com coeficiente de Poisson assumido como 0,35 (outros valores foram testados e não alteram a tendência do resultado final da análise), e espessuras e rigidezes relacionadas a seguir:

- Revestimento: 5,1cm e 3184MPa (para análise que utiliza MR);

○ Base: $38 \mathrm{~cm}$ e $443 \mathrm{MPa}$;

○ Subbase: $24 \mathrm{~cm}$ e $166 \mathrm{MPa}$;

- Subleito: $49 \mathrm{~cm}$ e $160 \mathrm{MPa}$;

- Temperatura $\left({ }^{\circ} \mathrm{C}\right.$ ): 25 (quando a temperatura não for o parâmetro em análise);

- Dados da curva mestra da mistura (Equação 8): $a=1,19 ; b=3,29 ; d=0,9130 ; g=0,51 ; \alpha 1=$ 0,$00121 ; \alpha 2=-0,1780 ; \alpha 3=3,0800$;

- Dado da curva de vida de fadiga por compressão diametral (CD): K1 = 3×10-11; K2 = -3,535 (Equação 5);

- Dados da curva de vida de fadiga por TD da mistura com CAP 65/90 e Tamanho Máximo Nominal (TMN) de 19,1mm: $\alpha=3,43 ; \mathrm{C} 11=0,001530 ; \mathrm{C} 12=0,528 ; \Delta=-1,211 ; \mathrm{Y}=3709564 ; \beta=-0,08$.

Tabela 1. Dados de temperatura do $\operatorname{ar}\left({ }^{\circ} \mathrm{C}\right)$ em uma Cidade do Nordeste e outra do Sul do país

\begin{tabular}{ccccccccccccc}
\hline \multicolumn{10}{c}{ Cidade do Nordeste do país } \\
\hline Mês & Jan & Fev & Mar & Abr & Mai & Jun & Jul & Ago & Set & Out & Nov & Dez \\
\hline 00:00 as 08:00h & 23,6 & 22,6 & 22,5 & 22,6 & 21,9 & 21,5 & 21,2 & 21,4 & 22,4 & 22,8 & 22,9 & 23,3 \\
08:00 as 16:00h & 33,8 & 32,3 & 31,4 & 31,1 & 31,2 & 31,6 & 33 & 34,8 & 35,8 & 35,9 & 35,6 & 35,5 \\
16:00 as 24:00h & 27,5 & 26,7 & 26,1 & 26 & 26 & 26 & 25,9 & 26,8 & 27,5 & 27,8 & 28,1 & 28,1 \\
\hline & & & & \multicolumn{1}{c}{ Cidade do Sul do país } & & & & & \\
\hline 00:00 as 08:00h & 16,4 & 16,3 & 15,4 & 12,8 & 10,2 & 8,4 & 8,1 & 9,2 & 10,8 & 12,5 & 14 & 15,4 \\
08:00 as 16:00h & 20,4 & 20,6 & 19,6 & 17,2 & 14,5 & 13,1 & 12,9 & 14,1 & 15 & 16,5 & 18,2 & 19,3 \\
$16: 00$ as 24:00h & 26,6 & 26,7 & 25,7 & 23,1 & 21,1 & 19,6 & 19,4 & 20,9 & 21,3 & 22,6 & 24,5 & 25,4 \\
\hline
\end{tabular}

As análises, cujo resumo encontra-se na Figura 5, demonstram os diferentes resultados de dano médio quando ocorre a variação de velocidade, temperatura e parâmetros de fadiga, que são associados a rigidezes específicas (fadiga por CD associado à MR; e fadiga por TD associada à |E*|). Os dados da Tabela 1 foram utilizados para demostrar a variação do dano com a variação de temperatura.

- De posse desses dados de entrada, foram realizadas as análises no CAP 3D-D, obtendo-se curvas de Dano médio x N, buscando-se verificar nos resultados obtidos: 
- Efeito da velocidade (Figura 5A), considerando iguais para todas as análises os dados de temperatura $\left(25^{\circ} \mathrm{C}\right),\left|\mathrm{E}^{*}\right|$ e TD. A escolha das velocidades teve como único intuito demonstrar os diferentes danos provocados ao pavimento, não tendo qualquer relação com os valores usualmente praticados no Brasil;

- Efeito da temperatura (Figura 5B), sendo, para isso, iguais para todas as análises os dados de velocidade $(60 \mathrm{~km} / \mathrm{h}),\left|\mathrm{E}^{*}\right|$ e TD;

- Efeito do parâmetro de fadiga (Figura 5C), considerando iguais para todas as análises os dados de velocidade $(60 \mathrm{~km} / \mathrm{h})$, temperatura $\left(25^{\circ} \mathrm{C}\right)$ e $\left|\mathrm{E}^{*}\right|$.

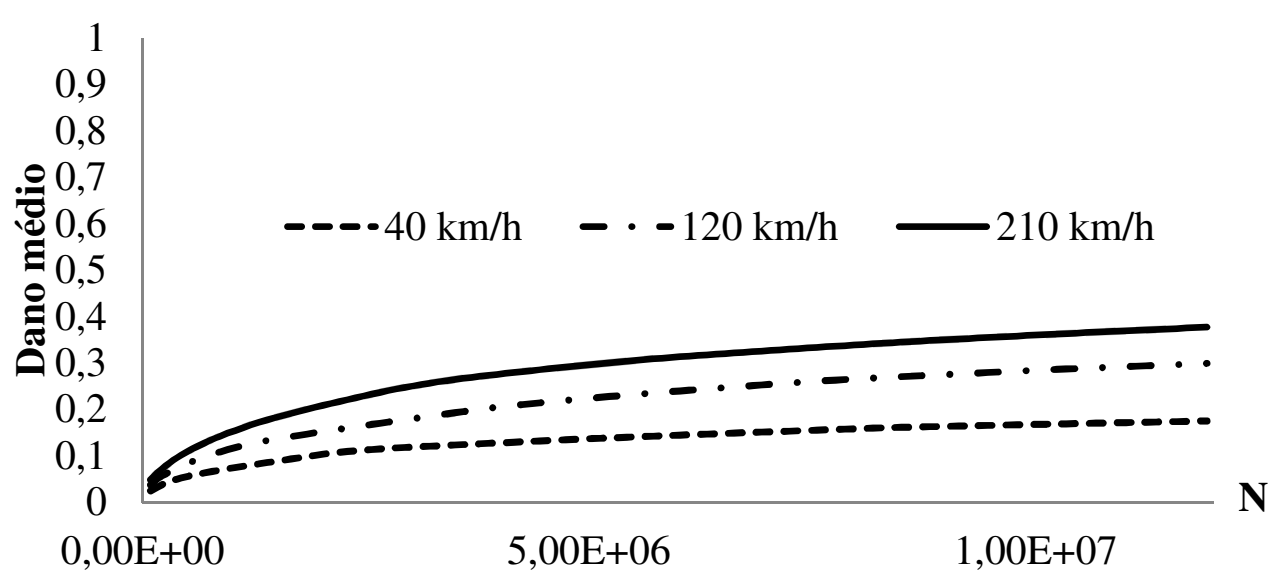

(a)

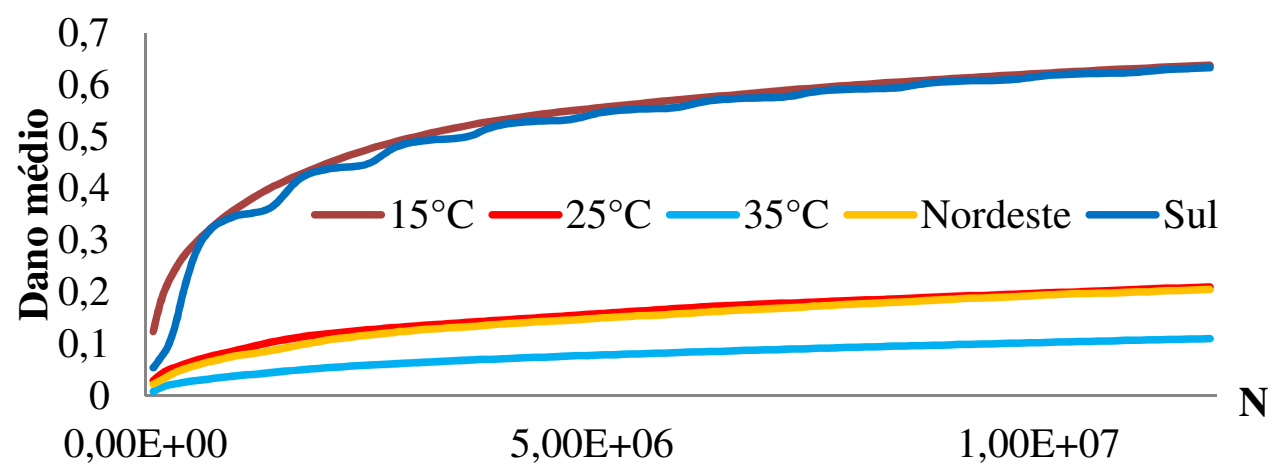

(b)

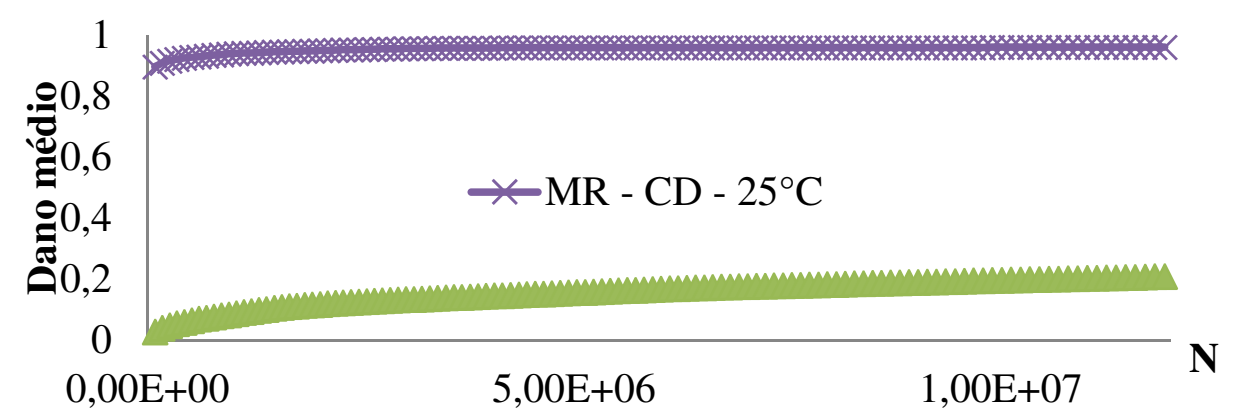

(b)

Figura 2. Dano médio x N: (a) Com variação de velocidade; (b) Com variação de temperatura; (c) Com variação dos parâmetros de fadiga 
A partir dessas curvas infere-se o seguinte:

- São diferentes, em uma mesma condição de análise estrutural e temperatura, os resultados de dano quando se utiliza diferentes velocidades. Quanto menor a velocidade, menor é o dano por fadiga ao longo do tempo, para a temperatura analisada $\left(25^{\circ} \mathrm{C}\right.$ no caso);

- São diferentes, em uma mesma condição de análise estrutural e velocidade, os resultados de dano quando em diferentes temperaturas. Quanto menor a temperatura, maior é o dano ao longo do tempo;

- Ao se analisar pavimentos em diferentes climas (e.g., Nordeste e Sul) há variação nos resultados de dano. A não inclusão da variação de temperatura levaria o projetista a considerar que uma mistura aplicada no Sul do país teria o mesmo comportamento que no Nordeste, o que percebe-se não ser verdade, sendo tal fato bastante relevante num método de alcance nacional;

- Numa mesma mistura é possível que surjam oscilações ao longo do tempo, como é o caso da análise para o Sul do país (Figura 5B). Essa variação, também encontrada no MEPDG e no LVECD, deve-se as altas amplitudes térmicas ao longo do ano, ao comportamento particular do CAP 65/90, i.e., elevada vida de fadiga e sensibilidade à temperatura, e ao fato do revestimento do exemplo ser delgado, o que o torna mais sensível ao dano;

- São diferentes, em uma mesma condição de análise estrutural, velocidade e temperatura, os resultados de dano quando se utiliza parâmetros de fadiga obtidos dos ensaios de CD ou TD. $\mathrm{O}$ dano provocado pelo $\mathrm{CD}$ é mais severo que o provocado pelo TD;

- Por fim, da Figura 5B, verifica-se, indiretamente, a importância de se utilizar o |E*|, uma vez que que temperaturas distintas geram resultados diferentes de dano, o que a consideração da rigidez em um única temperatura (por exemplo, $25^{\circ} \mathrm{C}$ ) não possibilita.

\section{CONCLUSÕES E RECOMENDAÇÕES}

Considerando o exposto, conclui-se que:

A frequência de carregamento exerce influência sobre o comportamento mecânico do material, haja vista a variação nos resultados de dano quando se modificou a velocidade nas análises;

A inclusão da variação de temperatura gera resultados de dano diferentes daqueles quando se considera uma temperatura fixa;

- Ainda com relação a variação de temperatura, percebe-se a importância da sua inclusão nas análises, principalmente quando se considera utilizar uma mesma mistura em diferentes regiões. 0 dano provocado na mistura se aplicada no Nordeste ou no Sul do Brasil é bastante diferente, ou seja, do ponto de vista de projeto a consideração da temperatura local leva a resultados de dano por fadiga regionais mais precisos do que fixa-la em um único valor nacional. É recomendado que se leve isso em conta num método de dimensionamento;

- Quando se compara velocidade e temperatura, a temperatura apresenta maior importância, principalmente se observadas as conclusões anteriores;

- A diferença nos resultados de simulação utilizando CD ou TD produz resultados distintos (CD mostrando-se mais severo), principalmente quando consideradas temperaturas diferentes. É importante investigar a eficiência dos dois resultados no intuito de encontrar aquele que fornece uma melhor correlação com os dados de campo;

- Por fim, a utilização do $\left|\mathrm{E}^{*}\right|$ se mostrou pertinente, dado que este parâmetro permite análises para diferentes condições de temperatura, o que na prática é importante para o dimensionamento de pavimentos considerando condições regionais num país de dimensões continentais.

A metodologia proposta mostra-se promissora, já que capturou os efeitos de velocidade e tempera- 
tura, além de trazer algumas questões sobre o efeito do ensaio de fadiga considerado. As análises contendo $\left|E^{*}\right|$ e TD combinam a premissa de steady state do pavimento, por meio da utilização de $\left|E^{*}\right|$ para avaliar as respostas estruturais (que variam com velocidade e temperatura), com o S-VECD. Trata-se, respectivamente, de um modelo para previsão de respostas combinado com um modelo de dano, numa metodologia two-step que pode facilitar o processo de predição de desempenho de pavimentos quanto a fadiga de materiais asfálticos em campo.

\section{REFERÊNCIAS}

Babadopulos, L. F. A. L.; J. B. Soares; V. T. F. Castelo Branco e F. V. Souza (2013) Aplicação de análogos mecânicos para representar o comportamento viscoelástico linear de misturas asfálticas e interpretar sua influência no módulo de resiliência. In: XVII Congresso Ibero-Latinoamericano del Asfalto, Guatemala.

Babadopulos, L. F. A. L.; J. B. Soares; V. T. F. Castelo Branco (2015) Interpreting fatigue tests in hot mix asphalt (HMA) using concepts from viscoelasticity and damage mechanics. Revista Transportes v. 23, n. 2, p. 85-94. ISSN: 2237-1346 (online). DOI: $10.14295 /$ transportes.v23i2.898.

Fritzen, M. A. (2016) Desenvolvimento e Validação de Função de Transferência para Previsão de Dano por Fadiga em Pavimentos Asfálticos. Tese (Doutorado em Engenharia Civil) COPPE/UFRJ. Rio de Janeiro.

Gouveia, B. C. S. (2016) Validação de modelos de previsão de módulo dinâmico para misturas asfálticas aplicadas em rodovias brasileiras. Dissertação (Mestrado em Engenharia de Transportes) - Centro de Tecnologia, Universidade Federal do Ceará, Fortaleza.

Holanda, A. S.; E. Parente Jr.; T. D. P. Araújo; L. T. B. Melo; F. Evangelista Jr. e J.B. Soares (2006). Finite Element Modeling of Flexible Pavements. XXVII Iberian Latin-American Congress on Computational Methods in Engineering (CILAMCE). Belém, Pará.

Huang, Y. H. (2004) Pavement Analysis and Design. 2ª edição. Ed. Pearson prentice Hall. ISBN: 0-13-142473-4. USA.

Kim, Y. R. (2009) Modeling of Asphalt Concrete. American society of Civil Engineers. Ed. Mc Graw Hill Construction. ISBN: 9780-07-146462-8. USA

Lemaitre, J. (1996) A Course on Damage Mechanics. 2aEdição. Ed. Springer. ISBN-13: 978-3540609803.

Mello, L. G. R.; M. M. Farias; E. S. Preussler e R. Preussler (2016) Análise do impacto do período de projeto de pavimentos no custo global de obras rodoviárias. Revista Transportes v. 24, n. 4, p. 64-74. ISSN: 2237-1346 (online). DOI: 10.14295/transportes.v24i4.1056.

Motta, L.M.G. (1991) Método de Dimensionamento de Pavimentos Flexíveis; Critério de Confiabilidade e Ensaios de Cargas Repetidas. Tese (Doutorado em Engenharia Civil). COPPE/UFRJ, Rio de Janeiro.

Nascimento, L. A. H. (2015) Implementation and Validation of the Viscoelastic Continuum Damage Theory for Asphalt Mixture and Pavement Analysis in Brazil. Tese (Doutorado Civil Engineering). North Carolina State University. Raleigh-USA.

NCHRP (2004) Guide for Mechanistic-Empirical Design of new and Rehabilitated Pavement Structures. Final Report. Part 3: Design Analysis. Chapter 3: Design of new and reconstructed flexible pavement. National Cooperative Highway Research Program - NCHRP. Illinois-USA.

NCSU (2014) Layered Viscoelastic Pavement Analysis for Critical Distresses - LVECD 1.0 Beta (User Guide). Department of Civil, Construction, and Environmental Engineering. North Carolina State University.

Shah, S. P.; S. E. Swartz e C. Ouyang (1995) Fracture Mechanics of Concrete: Applications of fracture Mechanics to Concrete, Rock and Other Quasi-Brittle Materials. Ed. Wiley Interscience. New York- USA. ISBN: 0-471-30311-9.

Torquato e Silva, S. A.; P. J. F. Vidal; A. S. Holanda e E. Parente Júnior (2013) Análise viscoelástica de pavimentos asfálticos utilizando elementos finitos e infinitos. Revista TRANSPORTES, v. 21, n. 3, p. 5-13. ISSN: 2237-1346 (online). DOI: 10.4237 /transportes.v21i3.676. 